\title{
Measures to Promote Adoption of Residential Photovoltaic Systems
}

\author{
Yoshihiro Yamamoto* \\ Department of Economics, Takasaki City University of Economics, Takasaki, Japan \\ * Corresponding author. Tel: +81 273447538, Fax: +81 273434840,E-mail: ysyama@tcue.ac.jp
}

\begin{abstract}
The purpose of this paper is to clarify the effects of a combination of electricity rates, the price of the electricity generated with a photovoltaic (PV) system, and a subsidy when a government aims at achieving a certain level of PV-system adoption. A microeconomic model based on classical demand theory is made. The case is mainly analyzed where the amount of PV-generated electricity is different from household to household while the amounts of electricity consumption and budget as well as utility functions are identical. Other cases are also mentioned. It is shown that a household prefers a higher PV-generated electricity price with a higher electricity rate to a higher subsidy if any one of the following conditions is satisfied with other things being equal: (1) it will have a relatively large amount of PV-generated electricity if it installs; (2) it has a relatively large amount of budget; or (3) it has a relatively small amount of electricity consumption. Furthermore, other things being equal, the difference in utility functions has no effect on the preference. This suggests, though the mixed effects of these conditions are not examined, that a combination optimal for a household does not always optimal for another.
\end{abstract}

Keywords: Residential Photovoltaic System, Feed-in tariff, Subsidy

\section{Introduction}

There will be several measures to promote adoption of residential photovoltaic (PV) systems. One is that a government subsidizes a household to install the system. Another one is that the electricity generated by a PV system is purchased by an electric utility. Moreover, the retail rate of electricity will influence a household's economic situation and affect the decision to install the system. Accordingly, three parameters, an electricity rate, a price at which the PVgenerated electricity is purchased by a utility, and a subsidy for installation, play a role in increasing the number of system-installed households. A government generally has control over these parameters. The purpose of this study is to obtain information available when a government sets them.

Most of existing studies addressing relevant issues are empirical or simulation ones calculating the value of PV systems or a break-even point, a combination of the parameters that makes it pay a household to install the system. For example, Mills et al. (2008) examine empirically the impact of electricity rate design on the economic value of PV systems for commercial customers in California. Carley (2009) shows using a two-part probit model that interconnection standards and RPS policies significantly increase the likelihood that a customer will adopt distributed generation capacity in the U.S. Rigter et al. (2010) determine the cost of PV system and obtain the optimal feed-in tariff by net present value analysis with Chinese data. Black (2004) shows that PV system installation is financially feasible under government incentives, net metering, high electricity rates, and other conditions in terms of rate of return, increase in property value, and cash flow.

These studies show the three parameters play an important role but do not deal with interrelations among them explicitly. This paper aims at filling this gap. I employ a different approach based on classical demand theory, in which consumers make decisions about purchases of goods by maximizing utility subject to budget constraints. Interestingly, to the best of my knowledge, the problem has not been investigated this way. I make a microeconomic model, examine efficient combinations of the parameters when a government 
intends to make a given number of households install the system, and analyze the payoffs of the relevant parties such as system-installed households, no-system households, and an electric utility.

I identify the locus of an electricity rate, a PV-generated electricity price, and a subsidy on which a government can make a given number of households install the system. The utility levels of a system-installed household and a no-system household are calculated and the utility maximization point for each household is identified. This will make some contribution to the discussion on how the electricity rate, the PV-generated electricity price, and the subsidy should be set from an equity point of view.

\section{Methodology}

A microeconomic model is set up. Since rational consumers will optimize decisions on purchasing PV systems based on their usual lifespan of 10-20 years, all quantities and monetary values employed in the model are set forth in terms of a fixed system lifespan.

Consider $N$ households with a market consisting of three goods: electricity, PV systems, and a composite of conventional goods. $N$ is sufficiently large. The government has a target of installed systems in $n$ of $N$ households.

Since the price elasticity of demand for electricity may be very low, we assume that if electricity rates change, household electricity consumption, $x$, will remain constant. Each installed PV system produces $e$ units of electricity. It is assumed that $x>e$.

All monetary value is normalized without loss of generality such that the price of the composite good is 1 . The price of a PV system, which includes the prices of PV generation equipment and installation, is denoted by $K$ and is constant.

In the model, a single electric utility, a government-regulated monopoly, supplies electricity to all households. Electricity rates may therefore be understood to be set by the government. For analytical simplicity, we assume the electricity rate to be a single, variable rate. Let $c$ represent the cost of generating a unit of electricity for the electric utility. Suppose the conventional electricity rate is set at $c$.

Let $y$ represent the budget of a household. The sum of the budgets of all households is denoted by $Y$. Funds for the subsidy are raised by taxation. It is assumed that a household must pay a tax according to its income, that is, the budget. Let $S$ be a subsidy for a household with an installed system. Then the tax rate for each household should be $n S / Y$.

Let the quantities of PV systems and composite goods purchased by a household be denoted by $q_{1}$ and $q_{2}$, respectively, with $q_{1}$ taking one of two values, 0 (no PV system installed) or 1 (PV system installed). Let the utility function be denoted by $u\left(q_{1}, q_{2}\right)$. We exclude utility obtained from electricity consumption. A household can do without a PV system since it can purchase all the electricity it consumes from the electric utility. Hence, it is plausible that the installation of a PV system can be valued in terms of finite quantities of the composite good. Thus, we define a function $v\left(q_{2}\right)$ such that the utility level at point $\left(0, q_{2}\right)$ is equal to that at point $\left(1, q_{2}-v\left(q_{2}\right)\right)$ on the $r$-p plane, i.e., $u\left(0, q_{2}\right)=u\left(1, q_{2}-v\left(q_{2}\right)\right)$. In other words, function $v\left(q_{2}\right)$ indicates the opportunity cost of installing the system in terms of the quantity of the composite good. It is reasonable to assume that $v\left(q_{2}\right)$ should satisfy $0 \leq v\left(q_{2}\right) \leq q_{2} \cdot v\left(q_{2}\right)$ is 
twice differentiable and that it holds that $0<v^{\prime}\left(q_{2}\right)<1$ and $v^{\prime \prime}\left(q_{2}\right)<0$. This implies that the larger the budget of a household, the higher the value of a PV system to that household, but the smaller the incremental value.

\section{Results}

There are many variables to be considered, $e, x$ and $y$ as well as $u\left(q_{1}, q_{2}\right)$ and $v\left(q_{2}\right)$. It is difficult to deal with them simultaneously, so we focus on $e$ in subsection 3.1 and then mention how to deal with the other variables in subsection 3.2.

\subsection{Different amounts of $P V$-generated electricity}

Suppose that household i's amount of PV-generated electricity is $e_{i}$. It is assumed that $e_{i}>e_{j}$ for all $i$ and $j$ such that $i<j$. It holds $N>\sum_{n} e_{i} / e_{n}$ since $N$ is sufficiently large. It is assumed that for any household $i, v(y-c x)<K-c e_{i}$ and $v\left(y-r_{0} x\right) \geq K-r_{0} e_{i}$ for some sufficiently large $r_{0}$. The meaning of this assumption, as shall become clear, is that if the electricity rate and PV-generated electricity price are both $c$ when $S=0$, no household will install PV systems, and that if both are $r_{0}$, all households will install systems.

The budget of household $i$ increases practically from $(1-n S / Y) y-r x$ to $(1-n S / Y) y-r x+S+p e_{i}$ if it installs a PV system. This is equivalent to the situation in which the budget remains at the same level $(1-n S / Y) y-r x$ while the price of PV systems decreases by $S+p e_{i}$. Hence, the budget constraint of household $i$ is $\left(K-S-p e_{i}\right) q_{1}+q_{2} \leq(1-n S / Y) y-r x$.

Since households maximize utility subject to the budget constraint, household $i$ installs the system if $v((1-n S / Y) y-r x) \geq K-S-p e_{i}$ and does not if $v((1-n S / Y) y-r x)<K-S-p e_{i}$. Therefore, the necessary and sufficient condition for exactly $n$ households to install the system is that the two inequalities $v((1-n S / Y) y-r x) \geq K-S-p e_{n}$ and $v((1-n S / Y) y-r x)<K-S-p e_{n+1}$ hold simultaneously. It will be shown step by step that there exists a combination $(r, p, S)$ that satisfies the following equation:

$v\left(\left(1-\frac{n S}{Y}\right) y-r x\right)=K-S-p e_{n}$

Eq. (1) guarantees that exactly $n$ households install the system.

\subsubsection{Controls of price and rates}

We first analyze a special case, where $S=0$. Let us make the arguable assumption that as the electricity rate rises, it becomes more favorable for a household to install a system and generate electricity itself, rather than purchase it if the PV-generated electricity is purchased at the electricity rate. In other words, it holds that $v(y-r x)-\left(K-r e_{i}\right)$ is increasing in $r$, i.e., $x v^{\prime}(y-r x)<e_{i}$.

Lemma 1. There exist $r_{1}$ and $p_{1}$ that uniquely satisfy Eqs. (2) and (3), respectively.

$$
\begin{aligned}
& v\left(y-r_{1} x\right)=K-r_{1} e_{n}, \\
& v(y-c x)=K-p_{1} e_{n} .
\end{aligned}
$$


Proof. These are shown from the assumptions $v(y-c x)<K-c e_{i}, \quad x v^{\prime}(y-r x)<e_{i}$, and $v\left(y-r_{0} x\right) \geq K-r_{0} e_{i}$.

Suppose the government chooses the lowest $r$ for any $p$ or the lowest $p$ for any $r$ to make exactly $n$ households install systems.

Lemma 2. The $r$ and $p$ set by the government must satisfy the following equation:

$v(y-r x)=K-p e_{n}$.

Proof. The curve defined by Eq. (4) on the $r$-p plane connects points $\left(c, p_{1}\right)$ and $\left(r_{1}, r_{1}\right)$, and is strictly upward-sloping (Fig. 1). Hence, there exists a unique solution $r$ for any $p$ that satisfies Eq. (4), and vice versa. A point on the curve represents the lowest $r$ for any $p$ or the lowest $p$ for any $r$ that satisfies the two inequalities.

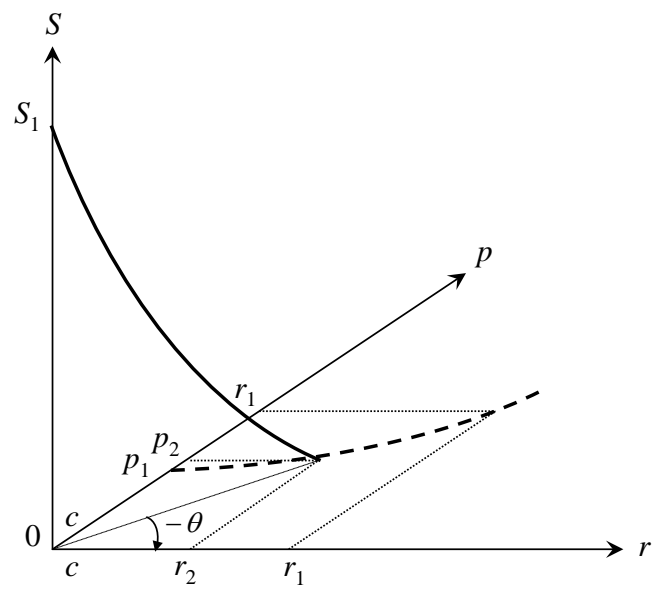

Fig. 1. The curves guaranteeing that exactly $n$ households install the PV system in the $r-p-S$ space. The dashed curve corresponds to the case where there is no subsidy. The solid curve corresponds to the case where a subsidy is introduced with the electric utility being compensated for the costs of purchasing PV-generated electricity.

The utility levels of system-installed household $i$ and a no-system household, and the profit of the electric utility, are as follows, respectively:

$$
\begin{aligned}
& u\left(1, y-r x-\left(K-p e_{i}\right)\right)(i=1, \cdots, n), \\
& u(0, y-r x), \\
& (r-c) N x-(p-c) \sum_{n} e_{i} .
\end{aligned}
$$

The government may make its decisions in determining $r$ and $p$ that the electric utility should be compensated for the costs of purchasing PV-generated electricity. Namely, the profit of the utility (7) is set at zero:

$$
(r-c) N x-(p-c) \sum_{n} e_{i}=0 .
$$


This forms a straight line containing point $(c, c)$ on the $r-p$ plane. Solving simultaneous equations (4) and (8) for $r$ and $p$ we obtain the solution $\left(r_{2}, p_{2}\right)$ shown in Fig. 1.

\subsubsection{Controls of subsidy for households}

Next we analyze another special case, where $r=p=c$.

Lemma 4. There exists a unique solution, $S_{1}$, that satisfies Eq. (1) when $r=p=c$. The government chooses $S_{1}$.

Proof. $v((1-n S / Y) y-c x)-\left(K-S-c e_{n}\right)$ is strictly increasing in $S$, and negative if $S=0$ and positive if $S=\left(r_{0}-c\right) Y x /\left(n y_{n}\right)$. Therefore, there exists a unique solution $S_{1}$.

\subsubsection{Controls of price, rates, and subsidy}

Now we return to a general case. Suppose that the electric utility is compensated for the costs of purchasing PV-generated electricity, that is, Eq. (8) holds.

Proposition 1. The curve defined by simultaneous Eqs. (1) and (8) is convex and connects points $\left(r_{2}, p_{2}, 0\right)$ and $\left(c, c, S_{1}\right)$ in the $r$ - $p$-S space (Fig. 1$)$. The government chooses the $r, p$, and $S$ on the curve.

Proof. See Appendix 1.

The utility levels of system-installed household $i$ and a no-system household are as follows, respectively:

$$
\begin{aligned}
& u\left(1,\left(1-\frac{n S}{Y}\right) y-r x-\left(K-S-p e_{i}\right)\right)(i=1, \cdots, n), \\
& u\left(0,\left(1-\frac{n S}{Y}\right) y-r x\right) .
\end{aligned}
$$

The combination $(r, p, S)$ that maximizes the utility of each household is obtained by maximizing the quantity of $q_{2}$ subject to simultaneous Eqs. (1) and (8). Let us investigate such a combination.

Proposition 2. For system-installed household $i$, the optimal combination is $\left(r_{2}, p_{2}, 0\right)$ if $e_{i} \geq \sum_{n} e_{i} / n$. Specifically, the optimal combination is $\left(r_{2}, p_{2}, 0\right)$ for household 1 . On the other hand, for system-installed household $n$ and no-system households, the optimal combination is $\left(c, c, S_{1}\right)$.

\section{Proof. See Appendix 2.}

Proposition 2 implies that if a household will have a relatively large amount of PV-generated electricity with a PV system, it prefers $\left(r_{2}, p_{2}, 0\right)$, while if it will have a relatively small amount of PV-generated electricity, it prefers $\left(c, c, S_{1}\right)$. 


\subsection{Modeling differences in budgets, electricity consumption, or utility functions}

First, suppose the amount of budget of household $i$ is $y_{i}$ while $x$ and $e$ are constant. To take into account that as a budget increases, installing a PV system becomes easier, we assume that $y_{i}>y_{j}$ for all $i$ and $j$ such that $i<j$. We can then do an analysis similar to the case considered above and show if a household has a relatively large budget, it prefers $\left(r_{2}, p_{2}, 0\right)$, while if it has a relatively small budget, it prefers $\left(c, c, S_{1}\right)$.

Next, suppose the amount of electricity consumed in household $i$ is $x_{i}$, where $x_{i}>e$, while $e$ and $y$ are constant. As the amount of electricity consumption increases, the budget for purchasing a PV system and the composite good decreases, and therefore installing a system becomes more difficult; to take this into account, we assume that $x_{i}<x_{j}$ for all $i$ and $j$ such that $i<j$. Then a similar analysis can be done and it is shown if a household has a relatively small amount of electricity consumption, it prefers $\left(r_{2}, p_{2}, 0\right)$, while if it has a relatively large amount of electricity consumption, it prefers $\left(c, c, S_{1}\right)$.

Lastly, we consider the case in which utility functions are different from household to household. Suppose household $i$ has a utility function $u_{i}\left(q_{1}, q_{2}\right)$, while $e, x$, and $y$ are constant. Assume $u_{i}\left(q_{1}, q_{2}\right)>u_{j}\left(q_{1}, q_{2}\right)$ for all $i$ and $j$ such that $i<j$ for any $\left(q_{1}, q_{2}\right)$ and define a function $v_{i}\left(q_{2}\right)$ such that $u_{i}\left(0, q_{2}\right)=u_{i}\left(1, q_{2}-v_{i}\left(q_{2}\right)\right)$ and $v_{i}\left(q_{2}\right)>v_{j}\left(q_{2}\right)$. The analysis in this case can also be done in the same way. In this case, it is shown that any combination $(r, p, S)$ guaranteeing exactly $n$ households install the PV system bring about the same level of utility to each household regardless of PV system installation.

\section{Conclusions}

In this paper, I have analyzed the relationship between an electricity rate, a PV-generated electricity price, and a subsidy to achieve a certain level of PV system installation, and examined the impact on the utility of households.

I found that a household prefers a higher PV-generated electricity price with a higher electricity rate to a higher subsidy if any one of the following conditions is satisfied with other things being equal: (1) it will have a relatively large amount of PV-generated electricity if it installs a PV system; (2) it has a relatively large amount of budget; or (3) it has a relatively small amount of electricity consumption. Furthermore, other things being equal, the difference in utility functions of households has no effect on the preference.

The results imply that welfare distribution varies depending on the parameter settings even if a fixed number of households install PV systems. This is because each household has its own amounts of PV-generated electricity, budget, and electricity consumption and utility function. Hence, it will be difficult to set parameters with which every household is satisfied. It then may be a policy option that a menu consisting of a combination of an electricity rate, a PVgenerated electricity price, and a subsidy for installation is offered to households. This may relieve unfairness to some extent.

In the model, each effect of the amounts of PV-generated electricity, budget, and electricity consumption and utility functions was investigated separately but the mixed effect of them was not. Investigating the mixed effects is very important particularly when a government practically determines the value of each parameter. An analytical approach used in this paper 
may be difficult to apply directly but the formulation can be used if, for example, a simulation method is used.

\section{References}

[1] A. Mills, R. Wiser, G. Barbose, and W. Golove, The impact of retail rate structures on the economics of commercial photovoltaic systems in California, Energy Policy 36, 2008, pp. 3266-3277

[2] S. Carley, Distributed generation: An empirical analysis of primary motivators, Energy Policy 37, 2009, pp. 1648-1659

[3] J. Rigter and G. Vidican, Cost and optimal feed-in tariff for small scale photovoltaic systems in China, Energy Policy 38, 2010, pp. 6989-7000

[4] A.J. Black, Financial payback on California residential solar electric systems, Solar Energy 77, 2004, pp. 381-388

\section{Appendix 1: Proof of Proposition 1}

The curve defined by simultaneous equations (1) and (8) connects $\left(r_{2}, p_{2}, 0\right)$ and $\left(c, c, S_{1}\right)$ in the $r-p$-S space. First, we show the curve is strictly downward-sloping. Define $\theta$ as the angle formed by the line (8) and the $r$-axis on the $r$-p plane (Fig. 1). Then it follows that $\cos \theta=\sum_{n} e_{i} / A$ and $\sin \theta=N x / A$, where $A=\sqrt{\left(\sum_{n} e_{i}\right)^{2}+(N x)^{2}}$. We are allowed to show the proposition with respect to the curve obtained by rotating the original curve define by (1) and (8) around the $S$-axis by $-\theta$. The obtained curve is on the $r$-S plane:

$$
v\left(\left(1-\frac{n S}{Y}\right) y-\left[c+(r-c) \frac{\sum_{n} e_{i}}{A}\right] x\right)=K-S-\left[c+\frac{N x}{A}(r-c)\right] e_{n} .
$$

The slope of the tangent is as follows:

$$
\frac{d S}{d r}=-\frac{\left[N e_{n}-v^{\prime}(r, S) \sum_{n} e_{i}\right] x / A}{1-(n / N) v^{\prime}(r, S)},
$$

where $v^{\prime}(r, S) \equiv v^{\prime}\left((1-n S / Y) y-\left[c+(r-c) \sum_{n} e_{i} / A\right] x\right)$.

This is strictly negative due to the assumption $N e_{n}>\sum_{n} e_{i}$, and thus the curve is strictly downward-sloping. Hence, there exists a unique solution $r$ for $S$ and vice versa that satisfies simultaneous equations (1) and (8). The government will choose a point on the curve since points on the curve represent the lowest $r$ for $S$ or the lowest $S$ for $r$ that satisfies the two inequalities.

It can be verified that the second-order differential $d^{2} S / d r^{2}$ is always positive since $v^{\prime \prime}\left(q_{2}\right)<0$. Hence, the curve is convex in the $r-p-S$ space.

\section{Appendix 2: Proof of Proposition 2}

We are allowed to prove Proposition 2 with respect to the rotated curve around the $S$-axis by $-\theta$. First, I deal with system-installed household $i$. The quantity of $q_{2}$ when $q_{1}=1$ is as follows: 
$\left(1-\frac{n}{N}\right) S+\frac{\left(N e_{i}-\sum_{n} e_{i}\right) x}{A}(r-c)+y-K-\left(x-e_{i}\right) c \quad(i=1, \cdots, n)$.

The points giving a fixed amount of $q_{2}$ form a straight line on the $r$-S plane. The quantity of $q_{2}$ is always increasing in $r$ due to the assumption $N e_{n}>\sum_{n} e_{i}$ and in $S$, too. The slope of the line giving a fixed amount of $q_{2}$ is obtained from (A3).

$$
\frac{d S}{d r}=-\frac{\left(N e_{i}-\sum_{n} e_{i}\right) x / A}{1-n / N} \quad(i=1, \cdots, n) .
$$

This is strictly negative due to the assumption $N e_{n}>\sum_{n} e_{i}$. The difference of the absolute values of slopes (A4) and (A2), which is denoted by $F_{i}(r, S)$, follows:

$$
\begin{aligned}
F_{i}(r, S) & \equiv\left[\frac{\left(N e_{i}-\sum_{n} e_{i}\right) x / A}{1-n / N}\right]-\left[\frac{\left(N e_{n}-v^{\prime}(r, S) \sum_{n} e_{i}\right) x / A}{1-(n / N) v^{\prime}(r, S)}\right] \\
& =\frac{x}{A} \cdot \frac{N e_{i}-\sum_{n} e_{i}-(N-n) e_{n}-\left(n e_{i}-\sum_{n} e_{i}\right) v^{\prime}(r, S)}{(1-n / N)\left[1-(n / N) v^{\prime}(r, S)\right]} .
\end{aligned} .
$$

The sign of $F_{i}(r, S)$ is positive if $e_{i} \geq \sum_{n} e_{i} / n$. Then, the optimal point is $\left(r_{2}, p_{2}, 0\right)$ for household $i$ since the utility is increasing both in $r$ and $S$. For household $n$, the optimal point is $\left(c, c, S_{1}\right)$ since $F_{n}(r, S)<0$. Note that if $i<j$, i.e., $e_{i}>e_{j}$, the absolute value of $d S / d r$ of household $i$ is larger than that of household $j$ from Eq. (4).

The proof for a no-system household is done similarly. We obtain the quantity of $q_{2}$ when $q_{1}=0$ as a function of $r$ and $S$ :

$-\frac{x \sum_{n} e_{i}}{A}(r-c)-\frac{n}{N} S+y-c x$.

This is always decreasing both in $r$ and $S$. The slope of the line giving a fixed amount of $q_{2}$ for a no-system household is obtained from (A6):

$\frac{d s}{d r}=-\frac{x \sum_{n} e_{i} / A}{n / N}$.

This is negative. The difference of the absolute values of slopes (A7) and (A2), which is denoted by $G(r, S)$, is as follows:

$$
\begin{aligned}
G(r, s) & \equiv\left[\frac{x \sum_{n} e_{i} / A}{n / N}\right]-\left[\frac{\left(N e_{n}-v^{\prime}(r, S) \sum_{n} e_{i}\right) x / A}{1-(n / N) v^{\prime}(r, S)}\right] . \\
& =\frac{x}{A} \cdot \frac{\sum_{n} e_{i}-n e_{n}}{(n / N)\left[1-(n / N) v^{\prime}(r, S)\right]}
\end{aligned}
$$

This is always positive. Therefore, the optimal point is $\left(c, c, S_{1}\right)$ since the utility is decreasing both in $r$ and $S$. 